\title{
0062 THE ROLE OF PUPILS LIAISONS ON TRAFFIC PENALTIES AND ROAD TRAFFIC INJURIES IN IRAN
}

E Ainy*, H Soori, A Montazeri, S Omidvari, A R Colonel Jahangiree, G R Shiran Correspondence: Safety Promotion and Injury Prevention research center of Shahid Beheshti University of Medical Sciences, Tehran, Iran

10.1136/ip.2010.029215.62

Objective The role of pupils liaisons education on social discipline promotion and road traffic injury prevention was the main objective of this study.

Methods It was a before-after interventional study on 2800 pupils randomly selected from six different districts of Tehran. Data was collected by a questionnaire for children's performances and other demographic information. Validity and reliability of questionnaire was determined by content validity and test re test.

Results Subjects were 2800 pupils 8-15 years (Mean and median of ages: 11 years). Overall, $47.7 \%$ of pupils were boys and others girls. In general, $85.9 \%$ of parents had positive reaction to point out of their filial, $11 \%$ with no reaction and only $3 \%$ were protested to filial pointing out. lack of seat belt use $39.1 \%$, speaking with mobile and driving $31.8 \%$ and speeding $29.8 \%$ as major offences. Recorded offences by traffic police before the intervention was 2789 cases ( $S D=2.6$ ). A significant differences with 17.9 percent reduction in offences were observed after intervention (2290 cases, $\mathrm{SD}=2.6, \mathrm{p}<0.001)$. In 9 part of education which was focused by pupils liaisons there was a range of (9.9-55.6\%) with significant reduction ( $p<0.001)$ except of sleepy or fatigue and mobile speaking during driving. The most offences reduction were eating and drinking during driving $(92.7 \%)$.

Conclusion Pupils' liaison program was effective on reduction of driving offences. The most causes of offences were eating or drinking during driving. All affairs which were educated by pupils liaisons were effective on offences reduction except mobile speaking and driving. 